\title{
Temporal evolution of the domain structure in a Poisson-Voronoi transformation
}

\author{
Eloi Pineda \\ Departament de Física i Enginyeria Nuclear, ESAB, Universitat Politècnica de \\ Catalunya, Av. Canal Olímpic s/n, Castelldefels, 08860 Barcelona, Spain. \\ E-mail: eloi.pineda@upc.edu
}

\section{Daniel Crespo}

Departament de Física Aplicada, EPSC, Universitat Politècnica de Catalunya, Av. Canal Olímpic s/n, Castelldefels, 08860 Barcelona, Spain.

\begin{abstract}
The temporal evolution of the domain size and free-boundary distributions is calculated for a Poisson-Voronoi transformation. In this kind of transformation a set of randomly-distributed domain seeds start growing simultaneously, all with equal isotropic growth rate, occupying the original untransformed space. At the end of the transformation, all the space is occupied and the final configuration is the well-known Poisson-Voronoi tessellation. In this work, the temporal evolution of the domain structure in a two-dimensional transformation is obtained by means of a calculation method recently presented [1]. The method is based on the differentiation of the domains by their number of extended collisions. It is found that the probability distribution of geometrical configurations for domains with a certain number of extended collisions is time-invariant all along the transformation. The calculation of these time-invariant probability distributions allows us to obtain the probability density function of any geometric characteristic of the domains at any finite time during the transformation, in this work this is applied to obtain the size and the free-boundary fraction distributions. As far as we know, it is the first time that an analytical solution is obtained for this system.
\end{abstract}

Keywords: Cellular solids (Theory), Coarse-graining (Theory), Random/ordered microstructures (Theory)

PACS numbers: 02.50.Ey, 81.30-t 


\section{Introduction}

\subsection{Domain structures generated in nucleation and growth transformations}

Nucleation and growth transformations are common in various scientific fields. In these transformations, stable new phase domains appear and grow into the metastable phase following certain nucleation and growth laws. During the growth, the domains interfere with each other because neighbouring domains compete for the available untransformed space between them. The result of a nucleation and growth transformation is usually an inhomogeneous system constituted by new phase domains and, in some cases, remaining untransformed regions between them. Many properties of these systems are determined by the structure of domains originated during the transformation. The nucleation and growth laws jointly with the characteristics of the interaction between the domains determine the overall kinetics of the transformation, and also the domain structure generated during the process.

The nucleation and growth laws can be characterized by a nucleation rate $I(X, t)$, which gives the density of nuclei appeared per unit time in the untransformed region, and a growth rate $u(X, t)$, which gives the growth velocity of the domain boundary into the untransformed phase. The nucleation and growth laws can be dependent on the time $t$ and on any other global or local property $X$. If they only depend on global properties of the system, then $X=X(t)$ and $I$ and $u$ become functions just of time $t$. In such case, we will say that the transformation has uniform nucleation and growth. Uniform nucleation and growth are expected in phase transformations where temperature, pressure or other properties of the system do not vary locally. The simplest interaction between domains is by direct impingement, when every domain grows occupying all the untransformed space until it collides with a growing neighbour. A static boundary is then formed at the points where the two domains collide and the two domains remain distinguishable. The domains can not grow into other stable domains and their growth is stopped when all the surrounding space has been already occupied by competing domains.

Transformations with direct impingement and diverse uniform nucleation and growth laws have been widely studied. In these transformations the interference between new phase domains is purely geometric, and the overall kinetics is described by the Kolmogorov-Johnson-Mehl-Avrami (KJMA) model[2][3][4][5] or adaptations of it. Transformations with KJMA kinetics are observed in many solid-solid and solid-gas reactions[6][7], they may take place in one, two or three dimensional spaces[8][9], and they include various kinds of physical processes like surface reaction processes[10][11], crystallization of amorphous semiconductor thin films[12] and glass crystallization[13][14][15].

The KJMA model is based on the calculation of the extended transformed fraction $\widetilde{x}(t)$, which is defined as the fraction of space occupied by the domains neglecting overlapping, that is the simple addition of the space occupied by all individual domains as if each of them was growig in isolation. In a space of dimension $D=2$, for example, 
the extended transformed fraction is written as

$$
\widetilde{x}(t)=\pi \int_{t^{\prime}=0}^{t^{\prime}=t} I\left(t^{\prime}\right) d t^{\prime}\left[\int_{t^{\prime \prime}=t^{\prime}}^{t} u\left(t^{\prime \prime}\right) d t^{\prime \prime}\right]^{2},
$$

where $\pi\left[\int_{t^{\prime \prime}=t^{\prime}}^{t} u\left(t^{\prime \prime}\right) d t^{\prime \prime}\right]^{2}$ is the size of a circular domain nucleated at time $t^{\prime}$. In the case of a uniform nucleation, uniform and isotropic growth rate and purely geometric impingement, the KJMA equation

$$
x(t)=1-\exp [-\widetilde{x}(t)]
$$

gives the temporal evolution of the actual space transformed fraction $x(t)$. As already stated, transformations following KJMA kinetics are expected in different physical systems; besides, diverse extensions of the KJMA equation have been proposed in order to extend its applicability to non-uniform nucleations[16][17][18][19], nonisotropic or non-uniform growth rates[20][21] and complex reactions with more than one subprocess[22].

In a transformation with known kinetics, the temporal evolution of the mean values of some of the domain structure characteristics can be calculated. In a KJMA transformation, for instance, if the size $a$ of a domain is defined as the length, area or volume occupied by a domain in one, two or three dimensional transformations, the mean size of the domains $\bar{a}$ is

$$
\bar{a}(t)=\frac{x(t)}{N(t)}
$$

where

$$
N(t)=\int_{t^{\prime}=0}^{t^{\prime}=t}[1-x(t)] I\left(t^{\prime}\right) d t^{\prime}
$$

is the overall density of domains at time $t$. Also the mean free-boundary fraction of the domains $\bar{b}$ can be calculated as

$$
\bar{b}(t)=1-x(t)
$$

where the free boundary fraction $b$ of a domain is defined as the fraction of the original domain boundary which is still in contact with untransformed phase. In a two-dimensional transformation, $b$ corresponds to the fraction of the original circular boundary which has not yet collided with other domains. In a KJMA transformation, where the nucleation is uniformly and randomly distributed, the value of $\bar{b}(t)$ corresponds to the probability of finding untransformed space $1-x(t)$.

Although the kinetics of KJMA transformations is thoroughly described by (2), the temporal evolution of the domain structure is not yet resolved. As far as we know, the probability distributions of size, free boundary, number of vertices or any other geometric characteristic of the domains have not been analytically obtained for transformations in spaces of dimension $D>1$. For the case of $D=1$, the domains are line segments that can be in just three collision states: 1) a segment without collisions, 2) a segment with one collision in one side and 3) a segment with collisions in both sides. 
The simplicity of the collision process in $D=1$ permits to calculate the probability of a domain of being in one of these three states at time $t$ and then, an analytical solution for the temporal evolution of the domain size distribution can be obtained. The temporal evolution of the domain size probability density function (PDF) is derived in [23][24][9] for one-dimensional transformations with various kinds of nucleation and growth laws. For $D=2$ and $D=3$ spaces, an analytical solution does not exist and the domain size distribution has been evaluated by means of different kinds of stochastic simulations, see for instance [25][26][27]. A set of evolution equations for the domain size distribution was previously derived by the authors[28][29], based on a semi-empirical approach. Apart from these approaches, the temporal evolution of the domain structure remains unresolved for transformations in $D>1$ spaces, even for the simplest nucleation and growth protocols.

At the end of a KJMA transformation all the space is occupied and the growth of the domains is stopped; the resulting domain configuration is a random subdivision or tessellation of space. Random tessellations of space are studied in many scientific areas[30][31][32], and in many cases they are indeed originated by a nucleation and growth process. Likewise as the temporal evolution of the domain structure, some average values of the final space tessellation can be calculated analytically[33], but the probability distributions of the domains geometrical characteristics remains equally unresolved.

\subsection{The Poisson-Voronoi transformation}

Maybe the simplest nucleation and growth transformation is the so-called "cell model" [24] or pre-existing nuclei transformation. In this transformation, a set of randomly distributed nuclei or "seeds" start growing simultaneously at $t=0$, all with equal growth rate $u(t)$ and with no further nucleation. Then, the nucleation law can be written as

$$
I(t)=\rho \delta(t)
$$

where $\rho$ is the density of seeds and $\delta($.$) is the Dirac delta function. The initial$ distribution of nuclei is a Poisson point distribution with density $\rho$ and all the domains have the same size and spherical geometry before impinging with each other. In this transformation, the direct impingement of two domains generates a flat boundary or surface between them, and so the initial spherical domains become progressively transformed into Voronoi polyhedra as the transformation advances. At the end of the transformation, each polyhedric domain comprise all the space nearer to the domain seed than to any other neighbouring seed; this final domain structure is the well-known Poisson-Voronoi (PV) tessellation[32]. For this reason, we will call this nucleation and growth process a PV transformation.

The kinetics of a PV transformation is described by the KJMA equation and the extended transformed fraction is easily calculated. For instance, in a 2-dimensional PV 
transformation with constant growth rate $u$

$$
\widetilde{x}(t)=\pi u^{2} t^{2} \rho .
$$

The mean domain size is $\bar{a}(t)=\frac{x(t)}{\rho}$ and the final value of $\bar{a}$ when $t \rightarrow \infty$ is obviously $\rho^{-1}$. A PV transformation is completely determined by $\rho$ and $u(t)$. Furthermore, with the appropiate time scaling, at a certain value of space occupation $x(t)$ the same geometrical configuration is obtained regardless of the specific growth rate function $u(t)$. In this work we will assume $u=1$, and so the radius of any domain without collisions will be equal to the time $t$.

The PDF of the final structure obtained in a PV transformation is known to be a gamma distribution [34][35]

$$
f^{S}(a) \propto a^{\nu-1} \exp (-\nu \rho a)
$$

where the exponent has values of $\nu=2,3.575$ and 5.586 for spaces with $D=1,2$ and 3 respectively[29]. The gamma distribution of sizes and the value of $\nu$ for PV tessellations was derived analytically for $D=1[24]$ and it was "empirically" calculated from computer simulations for $D=2$ and $D=3[34][35]$. Similarly, the temporal evolution of the PDF of domain sizes in a PV transformation is known only for $D=1$, and the temporal evolution of the domain structure during the transformation remains unsolved for PV transformations with $D>1$.

In this paper, we present an analytical calculation of the temporal evolution of the domain size and free boundary PDFs in a PV transformation. The calculation method and some results for the domain size PDF were presented in [1], here we present new results for the free-boundary fraction and details of the mathematical derivation. For the sake of simplicity, the calculation is developed for a PV transformation in a $D=2$ space, although the method is general and can be applied to a higher space dimensionality. Furthermore, the method can be extended to the calculation of the temporal evolution of any other geometric characteristic of the domain structure. The calculation is based on the probability of the domains to have a certain collision configuration; this probability is found to be time-invariant for the domains with a fixed number of collisions. As far as we know, it is the first time that this classical system is resolved analytically.

The derivation of the time-invariant probability distribution of collision configurations, which is the basis of the present calculation, is presented in section 2 . In section 3 and section 4 the previous result is used to calculate the PDF of the domains free boundary and size at any finite time during the transformation. In section 5, the possible extensions to other $D$ values and to the calculation of other geometric characteristics are discussed. The possibility of using a similar method for obtaining the temporal evolution of the domain structure in KJMA transformations with more complex nucleation and growth laws is also discussed. Finally, the potential applicability of the presented calculation is emphasized. 


\section{Time-invariant probability distribution of geometrical configurations}

The calculation method used in this work was presented in [1]. The method is based on the distinction of the domains by their number of "extended collisions". In a PV transformation with $u=1$, a domain nucleated at some point $O$ occupies all the surrounding space within a distance $t$ from $O$, provided that this space has not been previously occupied by a neighbouring domain. Two neighbouring domains collide if the distance between their nucleation points is smaller than $2 t$, and the space between them is not occupied by a third domain. The number of extended collisions of a domain with nucleation point $O$ is defined as the number of neighbouring domains with nucleation points $O_{i}$ nearer than a distance $2 t$ from $O$. Some of these extended collisions are real direct collisions that generate static rectilinear boundaries between the domains, while some other extended collisions are screened by previous collisions.

The probability of a domain to have $k$ extended collisions is then equal to the probability of finding $k$ nuclei at a distance from the domain origin smaller than $2 t$. In a two-dimensional PV transformation, the nucleation points constitute a Poisson distribution with density $\rho$ and this probability is then given by

$$
T_{k}(t)=\frac{\left(4 \pi t^{2} \rho\right)^{k} \exp \left(-4 \pi t^{2} \rho\right)}{k !}
$$

which is the probability of finding just $k$ nucleation points in a $4 \pi t^{2}$ area. It should be noted that the term $\exp \left(-4 \pi t^{2} \rho\right)$ is the probability of finding no nucleation points in such area, and it corresponds to the fraction of domains $T_{0}(t)$ that have not yet collided at time $t$. The temporal evolution of $T_{k}(t)$ with $k$ going from 0 to 9 is shown in figure 1. At $t=0$, all the domains have $k=0$ collisions and so $T_{0}(0)=1$ and $T_{k}(0)=0$ for $k>0$. Then, the number of domains with $k=0$ diminishes progressively as the transformation advances and the probability of finding not a single nucleation point in a $4 \pi t^{2}$ area becomes progressively lower. Of course, $T_{k}(t \rightarrow \infty)=0$ for any value of $k$ because there is a null probability of finding a finite number of nucleation points in an infinite area. Then, all the $T_{k}(t)$ functions with $k>0$ have a maximum at a certain intermediate time during the transformation and, as it is expected, the populations of domains with high $k$ number become more and more important at the end of the transformation. For instance, at a transformed fraction of $x\left(t_{0.5}\right)=0.5$, that is when half of the space is already occupied, $99 \%$ of the domains have $k \leq 7$ and the larger population correspond to $k=2$ with $T_{2}\left(t_{0.5}\right)=0.240$, while at $x\left(t_{0.95}\right)=0.95$ it is necessary to reach $k=21$ in order to encompass $99 \%$ of the domains and the larger $T_{k}(t)$ corresponds to $k=11$ with $T_{11}\left(t_{0.95}\right)=0.115$.

Each individual domain has a collision configuration at time $t$, which is determined by the set of nucleation point positions $\left\{O_{i}\right\}(i=1 . . k)$ of the $k$ domains that may collide with it, this collision configuration determines its size and geometry. In a $D=2$ space, these positions are easily expressed in a polar coordinates system centered in the nucleation point of the domain by a distance $2 t_{i}$ and an angle $\theta_{i}(i=1 . . k)$, with values of $t_{i}$ ranging from 0 to $t$, and $\theta_{i}$ ranging from 0 to $2 \pi$. As obtained in [1], the probability 
of finding a certain collision configuration $\left\{t_{1}, \theta_{1}, . ., t_{k}, \theta_{k}\right\}$ at time $t$ is

$$
P_{k}\left(t_{1}, \theta_{1}, . ., t_{k}, \theta_{k}, t\right)=4^{k} \rho^{k} \exp \left(-4 \pi t^{2} \rho\right) \prod_{i=1}^{k} t_{i} d t_{i} d \theta_{i},
$$

where $4 \rho t_{i} d t_{i} d \theta_{i}$ is the probability of finding a nucleation point at position $\left(2 t_{i}, \theta_{i}\right)$ and $\exp \left(-4 \pi t^{2} \rho\right)$ is the probability of finding no more nucleation points nearer than $2 t$. By a simple normalization $l_{i}=t_{i} / t,(10)$ leads to

$$
P_{k}\left(l_{1}, \theta_{1}, . ., l_{k}, \theta_{k}, t\right)=T_{k}(t) \frac{k !}{\pi^{k}} \prod_{i=1}^{k} l_{i} d l_{i} d \theta_{i},
$$

where $l_{i}$ can have values ranging from 0 to 1 . Each particular set $\left\{l_{i}, \theta_{i}\right\}$ determines a collision configuration; figure 2 shows one possible collision configuration for the case $k=4$. In the figure, the extended collision $k=3$ is screened by collision $k=1$. It is clear that, if at time $t$ the set $\left\{l_{1}, \theta_{1}, . ., l_{k}, \theta_{k}\right\}$ is known for a domain, then it is possible to calculate any of its geometric properties. In deriving (10) and (11) we assumed that $t_{i-1}<t_{i}<t_{i+1}$, if this temporal order of the collisions was not considered the probabilities should be divided by $k$ !.

The probability distribution of (11) is composed by the probability of finding a domain with $k$ collisions at time $t$, that is $T_{k}(t)$, and the time-invariant probability

$$
\frac{k !}{\pi^{k}} \prod_{i=1}^{k} l_{i} d l_{i} d \theta_{i}
$$

of finding a collision configuration $\left\{l_{1}, \theta_{1}, . ., l_{k}, \theta_{k}\right\}$ among the the domains with $k$ collisions. In the next two sections, we will show how these time-invariant probabilities of geometrical configurations can be used for calculating the PDFs of free boundary and size of the domains at any finite time $t$ during the transformation.

\section{Free boundary distribution}

A time-invariant probability distribution of collision configurations implies a timeinvariant probability distribution of domain free-boundary fraction $b$. That is, the probability of finding a domain with a given value of $b$ among the population of domains with a certain $k$ number is constant all along the transformation, while the temporal evolution of the overall probability distribution is then given by the evolution of the domain populations $T_{k}(t)$. In this section, (9) and (12) are used for calculating the PDF of free boundary fraction $f^{B}(b, t)$ in a two-dimensional PV transformation.

In a $D=2$ space the domains have an initial circular boundary and each collision

at a distance $l_{i}$ occupies a fraction $\frac{\arccos \left[l_{i}\right]}{\pi}$ of this circular perimeter, as is illustrated in figure 2. However, in order to calculate the free-boundary fraction of a domain the overlappings between different collisions should be taken into account. The angular segment of the domain perimeter occupied by a collision at $\left(l_{i}, \theta_{i}\right)$ comprise all the angles between the limiting points

$$
\alpha_{l i}=\theta_{i}-\arccos \left[l_{i}\right], \quad \alpha_{r i}=\theta_{i}+\arccos \left[l_{i}\right] .
$$


Hence, the function

$$
\begin{aligned}
C_{i}\left(l_{i}, \theta_{i}, \alpha\right)= & 1-H\left(\alpha-\alpha_{l i}\right) H\left(\alpha_{r i}-\alpha\right)-H\left(\alpha-\alpha_{l i}-2 \pi\right)- \\
& -H\left(\alpha_{r i}-\alpha-2 \pi\right),
\end{aligned}
$$

where $H($.$) is the Heaviside step function, gives a value of 1$ for any angle out of the segment $\left[\alpha_{l i}, \alpha_{r i}\right]$ and a value of 0 for any angle inside the segment. The previous expression allows to build a general function for calculating the free-boundary fraction of a domain, which can be written as

$$
B_{k}\left(l_{1}, \theta_{1}, . ., l_{k}, \theta_{k}\right)=\frac{1}{2 \pi} \int_{0}^{2 \pi} \prod_{i=1}^{k} C_{i}\left(l_{i}, \theta_{i}, \alpha\right) d \alpha .
$$

Functions $B_{k}$ calculate the value of $b$ for a domain with $k$ extended collisions and a certain collision configuration $\left\{l_{1}, \theta_{1}, . ., l_{k}, \theta_{k}\right\}$. Obviously,

$$
B_{1}\left(l_{1}, \theta_{1}\right)=1-\frac{\arccos \left[l_{1}\right]}{\pi}
$$

as it is expected.

Now, the time-invariant PDF of free boundary fraction, $g_{k}^{B}(b)$, for the domains with $k$ extended collisions can be calculated using the $B_{k}$ functions and the time-invariant probability of (12). The first of these PDFs, corresponding to $k=0$, is easily obtained as

$$
g_{0}^{B}(b)=\delta(b-1),
$$

because the domains without collisions maintain the original circular boundary and so they all have $b=1$. For the case of $k \geq 1$ the calculation can be performed by means of

$$
\begin{array}{r}
g_{k}^{B}(b) d b=\frac{k !}{\pi^{k}} \int_{l_{1}=0}^{1} \int_{\theta_{1}=0}^{2 \pi} \ldots \int_{l_{k}=l_{k-1}}^{1} \int_{\theta_{k}=0}^{2 \pi} \delta\left(b-B_{k}\left(l_{1}, \theta_{1}, . ., l_{k}, \theta_{k}\right)\right) \prod_{i=1}^{k} l_{i} d l_{i} d \theta_{i}= \\
=\frac{1}{\pi^{k}} \int_{l_{1}=0}^{1} \int_{\theta_{1}=0}^{2 \pi} \ldots \int_{l_{k}=0}^{1} \int_{\theta_{k}=0}^{2 \pi} \delta\left(b-B_{k}\left(l_{1}, \theta_{1}, . ., l_{k}, \theta_{k}\right)\right) \prod_{i=1}^{k} l_{i} d l_{i} d \theta_{i} .
\end{array}
$$

For small $k$ numbers the previous integration can be solved analytically, and a numerical integration can be always performed for any value of $k$. For $k=1$ the integration with $\delta\left(b-B_{1}\left(l_{1}, \theta_{1}\right)\right)$ is equivalent to a simple variable change $\cos (\pi(1-b))=l_{1}$, and taking into account that the limits $0<l_{1}<1$ imply that $0.5<b<1$ we obtain

$$
g_{1}^{B}(b)=\pi \sin (2 \pi(1-b)) H(b-0.5)
$$

For $k=2$ the analytical solution become more complex giving a more awkward result

$$
\begin{aligned}
g_{2}^{B}(b)=\frac{1}{2}[1 & \left.+\frac{\pi b}{2} \sin (2 \pi(1-b))-\left(1+\pi^{2} b^{2}\right) \cos (2 \pi(1-b))\right]+ \\
& +H(b-0.5)\left[\frac{3 \pi}{2}(1-b) \sin (2 \pi(1-b))\right]+ \\
& +H(b-0.5)\left[\pi^{2} b\left(b-\frac{1}{2}\right) \cos (2 \pi(1-b))-\sin ^{2}(2 \pi(1-b))\right] .
\end{aligned}
$$


In this work, for larger $k$ numbers the integration of (20) was performed numerically using a Monte Carlo integration method. Figure 3 shows the calculated $g_{k}^{B}(b)$ functions with $k$ numbers going from 1 to 9 ; the numerical integration was performed assuring a relative error lower than $10^{-3}$. It should be noticed that the probability of finding domains with $b=0$ is not null for $k>2$, because a fraction of the domains with 3 or more extended collisions may be completely blocked by their neighbours. So, the $g_{k}^{B}(b)$ functions with $k \geq 3$ are composed by a continous function within the interval $(0,1]$, which is shown in figure 3, and a dirac delta function at $b=0$. The area of the dirac delta function corresponds to the probability of finding a domain with $b=0$ among the population of domains with $k$ extended collisions; the values of this probability are shown in figure 4 for different $k$ numbers. It is interesting to note that the fraction of domains with the whole boundary in contact with neighbouring domains, and so already transformed into static Voronoi polygons, is surprisingly low even for large $k$ numbers. For instance, only $40 \%$ of the domains with 10 extended collisions are completely blocked by their neighbours.

Now, the temporal evolution of the overall PDF $f^{B}(b, t)$ can be obtained as

$$
f^{B}(b, t)=\sum_{k=0}^{\infty} g_{k}^{B}(b) T_{k}(t),
$$

that is adding the different contributions of the time-invariant $g_{k}^{B}(b)$ at a certain time $t$. When the transformation starts, at $x(t)=0$, all the domains have $b=1$ and $f^{B}(b, t)=\delta(b-1)$. As the transformation advances the domains suffer collisions and the probability of finding a domain with a high free-boundary fraction is reduced. Figure 5 shows $f^{B}(b, t)$ calculated at $x(t)=0.05,0.25,0.5$ and 0.75 . In each distribution the domains with $k=0$ contribute as a dirac delta function $T_{0}(t) \delta(b-1)$ at $b=1$. On the opposite side of the plot, the fraction of domains completely blocked contribute with another delta function at $b=0$, which increases its area as the transformation advances. For instance, the distribution at $x\left(t_{0.05}\right)=0.05$ is composed basically by $T_{0}\left(t_{0.05}\right)=0.815, T_{1}\left(t_{0.05}\right)=0.167$ and $T_{2}\left(t_{0.05}\right)=0.017$, and practically a null probability of finding a domain with $b=0$. At higher values of $x(t)$ the contribution of domains with larger $k$ becomes more important and the PDF of free-boundary fraction is moved towards lower values of $b$, at $x\left(t_{0.75}\right)=0.75$ the main population of domains is $T_{5}\left(t_{0.75}\right)=0.171$, the fraction of domains with $b=0$ have reached a value of 0.097 and the overall PDF is calculated adding the contributions of the domains with $k \leq 12$, which constitute more than $99 \%$ of the total structure. Figure 6 shows the temporal evolution of the fraction of domains with $b=1$ and $b=0$ between $x(t)=0$ and $x(t)=0.95$, as already noted, the fraction of domains completely blocked is surprisingly low even for relatively high values of the transformed fraction.

The accuracy of the result obtained for $f^{B}(b, t)$ at a certain time $t$ depends on the number of $k$-contributions used. The calculation of the $g_{k}^{B}(b)$ functions can be realized for any finite number of $k$, but the numerical integration time increases as $2^{k}$ for a fixed accuracy. Therefore, although in principle $f^{B}(b, t)$ can be calculated to any 
desired accuracy at any finite time, the calculation of (25) becomes impractical at the end of the transformation $(x(t) \rightarrow 1)$ as it is necessary to add an infinite number of $k$-contributions. However, at that moment all the domains tend to have $b=0$, and so the overall PDF becomes $f^{B}(b, t \rightarrow \infty)=\delta(b-0)$. Figure 7 shows the comparison of the result obtained in the present calculation at $x(t)=0.5$ with the result obtained in stochastic simulations of a PV transformation. These simulations were performed in $2048 \times 2048$ grids with periodic boundary conditions. In the simulations the domain seeds were randomly distributed and the seed density was chosen to ensure a number of domains not less than $10^{2}$; the domains grow with a constant velocity and each grid point is assigned to the domain that reaches it first. The result shown in figure 7 (bars) correspond to the average distribution obtained from 100 of such simulations. More details of the simulations were presented elsewhere [28]. The comparison with stochastic simulations were performed in order to check the validity of the above calculations; furthermore, figure 7 shows that the analytical calculation gives a much more detailed information of the free-boundary distribution than the stochastic simulations.

One interesting result connected with the present development is the calculation of the mean free-boundary fraction $\bar{b}_{k}$ of the domains with $k$ extended collisions. Obviously the mean value of a time-invariant distribution is a constant value, which can be shown to be

$$
\bar{b}_{k}=\left(\frac{4-1}{4}\right)^{k}
$$

The derivation of this result was presented in [1], and it means that each extended collision provokes an average reduction of a $1 / 4$ of the domains free-boundary fraction. Furthermore, the overall mean free-boundary fraction of the domains at a certain time $t$ is then

$$
\bar{b}(t)=\sum_{k=0}^{\infty} \bar{b}_{k} T_{k}(t)=\sum_{k=0}^{\infty}\left(\frac{4-1}{4}\right)^{k} T_{k}(t)=\exp \left(-\pi t^{2} \rho\right) .
$$

The term $\pi t^{2} \rho$ in the right-hand term of the previous equation is the extended transformed fraction $\widetilde{x}(t)$ for a two-dimensional PV transformation given in (7). Hence, recalling (2) and (5), the previous equation corresponds to the KJMA equation for this transformation. The previous result can be extended to $D=1$ and $D=3$ dimensions giving $\bar{b}_{k}=\left(\frac{2^{D}-1}{2^{D}}\right)^{k}$, and this also leads to the KJMA equation for such systems.

\section{Size distribution}

A collision configuration $\left\{l_{i}, \theta_{i}\right\}$ determines unequivocally a domain size. In this section we use (9) and (12) to calculate the domain-size PDF, $f^{S}(a, t)$, of a two-dimensional PV transformation. In a $D=2$ space, the size $a$ of a domain is defined as the area occupied by the domain at time $t$, in order to perform a similar development to the one in the previous section, a normalized size of the domains is defined as

$$
s=\frac{a}{\pi t^{2}} \text {. }
$$


Therefore, a domain without collisions, and so maintaining its original circular shape, has $s=1$; a domain with one collision must have $0.5<s<1$; and domains with more than one collision must have $0<s<1$. Now, the time-invariant PDFs $g_{k}^{S}(s)$ of normalized sizes of the domains with a given $k$ number can be calculated in a similar way as the free-boundary fraction PDFs of the previous section.

The first of these size PDFs, corresponding to $k=0$, is easily obtained as

$$
g_{0}^{S}(s)=\delta(s-1),
$$

because all the domains without collisions have $s=1$ or equivalently a size $a=\pi t^{2}$. In order to calculate the size PDF for domains with $k \geq 1$, a general expression for the domain size in function of the collision configuration has to be obtained. Substituting $l_{i}$ by $\frac{l_{i}}{r}$ in equations (13) and (15) the function

$$
C_{i}^{S}\left(\frac{l_{i}}{r}, \theta_{i}, \alpha\right)=\left\{\begin{array}{cc}
C_{i}\left(\frac{l_{i}}{r}, \theta_{i}, \alpha\right), & \frac{l_{i}}{r}<1 \\
1, & \frac{l_{i}}{r}>1
\end{array}\right.
$$

can be defined. A normalized domain with a collision at $\left(l_{i}, \theta_{i}\right)$ is depicted in figure 8 . By using polar coordinates $(r, \alpha)$ with origin at the nucleation point of the domain $O$, the function $C_{i}^{S}\left(\frac{l_{i}}{r}, \theta_{i}, \alpha\right)$ gives a value of 1 at any point inside the grey zone of the figure and 0 elsewhere. Integration of function $C_{i}^{S}\left(\frac{l_{i}}{r}, \theta_{i}, \alpha\right)$ over all the points going from $r=0$ to $r=1$ and $\alpha=0$ to $\alpha=2 \pi$ gives the grey area in figure 8 .

Using function $C_{i}^{S}$, a general function $S_{k}\left(l_{1}, \theta_{1}, . ., l_{k}, \theta_{k}\right)$, which computes the normalized size of a domain with a given $\left\{l_{1}, \theta_{1}, . ., l_{k}, \theta_{k}\right\}$ configuration taking into account the overlappings between collisions, can be written as

$$
S_{k}\left(l_{1}, \theta_{1}, . ., l_{k}, \theta_{k}\right)=\frac{1}{\pi} \int_{r=0}^{r=1} \int_{\alpha=0}^{\alpha=2 \pi} \prod_{i=1}^{i=k} C_{i}^{S}\left(\frac{l_{i}}{r}, \theta_{i}, \alpha\right) r d r d \alpha .
$$

Obviously, for $k=1$ the previous integration gives

$$
S_{1}\left(l_{1}, \theta_{1}\right)=1-\pi^{-1}\left[\arccos \left(l_{1}\right)-l_{1}\left(1-l_{1}^{2}\right)^{\frac{1}{2}}\right]
$$

as expected.

Now, the computation of $g_{k}^{S}(s)$ for $k \geq 1$ can be performed by means of

$$
\begin{array}{r}
g_{k}^{S}(s) d s=\frac{k !}{\pi^{k}} \int_{l_{1}=0}^{1} \int_{\theta_{1}=0}^{2 \pi} \ldots \int_{l_{k}=l_{k-1}}^{1} \int_{\theta_{k}=0}^{2 \pi} \delta\left(s-S_{k}\left(l_{1}, \theta_{1}, . ., l_{k}, \theta_{k}\right)\right) \prod_{i=1}^{k} l_{i} d l_{i} d \theta_{i}= \\
=\frac{1}{\pi^{k}} \int_{l_{1}=0}^{1} \int_{\theta_{1}=0}^{2 \pi} \ldots \int_{l_{k}=0}^{1} \int_{\theta_{k}=0}^{2 \pi} \delta\left(s-S_{k}\left(l_{1}, \theta_{1}, . ., l_{k}, \theta_{k}\right)\right) \prod_{i=1}^{k} l_{i} d l_{i} d \theta_{i} .
\end{array}
$$

Likewise the calculation of the free-boundary distributions in the previous section, a numerical integration of (34) using a Monte Carlo method is possible for any value of $k$. Figure 9 shows the calculated $g_{k}^{S}(s)$ for $k$ going from 1 to 9 . Similarly to the freeboundary case $g_{1}^{S}(s)=0$ for $s<0.5$, because a domain with only one collision have at least one half of its initial circular shape. As seen in figure 9, the mean value of $s$ given 
by the $g_{k}^{S}(s)$ functions decreases progressively as the number $k$ of extended collisions increases and, similarly to the free-boundary fraction case, each population of domains with a certain number of collisions have a constant mean value of normalized size.

From the time-invariant $g_{k}^{S}(s)$ functions, the total PDF of normalized sizes at a certain time $t$ can be calculated as

$$
g_{\text {total }}^{S}(s, t)=\sum_{k=0}^{\infty} g_{k}^{S}(s) T_{k}(t),
$$

and then the total size PDF is obtained using the variable change in (28), that is

$$
f^{S}(a, t)=g_{\text {total }}^{S}(s, t) \frac{d s}{d a} .
$$

Figure 10 shows the temporal evolution of this size PDF. Black dashed lines correspond to the calculated $f^{S}(a, t)$ at overall transformed fractions of $x(t)=0.05,0.25,0.5$ and 0.75 . Grey line shows the final gamma distribution of (8) corresponding to $x(t)=1$. The calculation of the integrals in (34) can be performed with any desired accuracy, so the $f^{S}(a, t)$ PDF can be calculated with the same accuracy adding the contributions of the necessary $k$-populations at a certain finite time $t$. As previously noted in section 3 , the estimation of the overall distribution at $t \rightarrow \infty$ would require to extend the calculation over an infinite number of $k$ values, but then the system tends to the configuration of the widely-studied PV tessellation[36][37][38]. This means that the size PDF at the final stages of the transformation tends to the gamma distribution of (8). Figure 11 shows the calculated size PDF at a time where the overall transformed fraction is $x(t)=0.5$; there the $f^{S}(a, t)$ calulated from (29), (34) and (35) is compared with the size distribution

obtained in stochastic simulations of the process. Details of the stochastic simulations were given in the previous section.

\section{Discussion and conclusions}

In a PV transformation the space is occupied by domains growing from randomly distributed nuclei, and this process generates a structure or network of domains. The fraction of occupied space decays exponentially with time and at $t \rightarrow \infty$ the structure becomes a PV tessellation. The calculation method presented in this manuscript allows, for the first time, to calculate analytically the probability distributions of the geometric properties of this structure at any finite time. The method has been applied to a transformation in a $D=2$ space, but it can be easily extended to other space dimensions. The time-invariant probability of a collision configuration in $D=1$ and $D=3$ systems can be written as

$$
\begin{array}{ll}
k ! \prod_{i=1}^{k} l_{i} d l_{i}, & \text { for } D=1 \\
k !\left(\frac{3}{4 \pi}\right)^{k} \prod_{i=1}^{k} l_{i}^{2} \sin \left(\theta_{i}\right) d l_{i} d \theta_{i} d \varphi_{i}, & \text { for } D=3
\end{array}
$$


where the position $O_{i}$ of a neighbouring nuclei is determined by $l_{i}$ for $D=1$ and by $\left(l_{i}, \theta_{i}, \varphi_{i}\right)$ for $D=3$. The previous expressions are equivalent to (12) for the $D=2$ case. Therefore, it is expected that the probability density function of a domain geometrical characteristic in $D \neq 2 \mathrm{PV}$ transformations can be obtained following a similar development to the one shown in section 3 and section 4 .

The results in section 3 and section 4 show the capacity of the method for calculating two of the domain properties: the size and the free-boundary fraction. Other properties of interest may be the number of domain vertices and the number or size of boundaries (in $D=2$ ) or faces (in $D=3$ ) between the domains. In polycrystalline materials, for example, grain boundaries influence atomic diffusion and they can also determine mechanical and thermodynamical properties of the system. It is reasonable to assume that equivalent functions to the $B_{k}$ and $S_{k}$ functions detailed in (16) and (31) can be defined for these other geometric properties and, therefore, similar calculations to the ones presented here could be performed in order to obtain their probability density functions. Moreover, a collision configuration determines unequivocally a domain shape and so a self-correlation function for that certain shape. Hence, the method presented here could be also used to calculate overall correlation functions of the system, or some linked properties like the small angle X-ray (SAXS) or neutron (SANS) scattering spectra[39]. SAXS and SANS are commonly used techniques for evaluating the grain size distribution in partially crystallized materials.

The main limitation for the application of the presented method is the numerical integration of (20) and (34). It should be noted that the integrals have to be performed just once for each $k$, as the resulting PDFs are time-invariant. However, as the time $t$ approaches infinity, an increasing number of $k$ distributions are needed in order to construct the overall PDF with a certain value of accuracy. In a $D=1 \mathrm{PV}$ transformation, the simplicity of the integrals allows to obtain explicit solutions and the infinite series sum corresponding to (25) or (35) has an explicit solution. Further work is needed to see if a similar development could be used in $D>1$ transformations. In addition, solving this issue could demonstrate the validity of a gamma distribution for the domain-size distribution of a PV tessellation, that, as far as we know, it is generally assumed but still not mathematically demonstrated.

At this point it is worth mentioning that underlying the presented method it is the fact that collision configurations occurring at different times are self-similar. This fact would be observed if pictures of collision configurations at different times were superimposed with adequate scaling and rotation, and becomes clearly uncovered after the normalization performed in (11). This is in fact the reason why the spatial and temporal dependences of the geometrical characteristics can be separated.

The Poisson-Voronoi transformation is just the simplest nucleation and growth process in which a structure or network is generated by means of the growth of randomly distributed nuclei. In many physical systems, the nucleation law is more complex and the presented calculation can not be applied. However, it seems probable that the presented method could be adapted to other nucleation protocols. In a previous work 
by the authors [40], the final structure generated in transformations with arbitrary time-dependent nucleation protocols was approximated assuming a PV-like domain-size distribution for the populations of domains nucleated simultaneously at each instant during the transformation. The present results allow the extension of that work to the calculation of the temporal evolution of the domain structure.

Finally, it should be noted that the present model contains the KJMA equation in itself. In fact, it is shown that the KJMA equation is a result of the time-invariant free-boundary distributions of the domains with a given $k$ number. This suggest that the presented method could be extended to any nucleation and growth transformation following KJMA kinetics, then covering a large number of systems with physical interest.

\section{Acknowledgments}

This work was funded by CICYT, Grant No. MAT2004-01214, and Generalitat de Catalunya, Grant No. 2005SGR00535. 


\section{References}

[1] Pineda E, Garrido V and Crespo D 2007 Physical Review E. 75 040107(R)-4.

[2] Avrami M 1939 Journal of Chemical Physics 7 1103-12

[3] Avrami M 1940 Journal of Chemical Physics 8 212-24

[4] Johnson W A and Mehl R F 1939 Trans. AIME 135 416-58

[5] Kolmogorov A N 1937 Bull. Acad. Sci. USSR, Phys. Ser. 1355-66

[6] Christian J W 1975 The theory of transformation in metal alloys, part I-Equilibrium amd general kinetic theory, 2nd Edition (New York: Pergamon press)

[7] Korobov A 1998 Journal of Mathematical Chemistry 24 261-90

[8] Ben-Naim E and Krapivsky P L 1996 Physical Review E 54 3562-68

[9] Yu G and Lai J K L 1995 Journal of Applied Physics 78 5965-69

[10] Brill M, Bloch J and Mintz M H 1998 Journal of alloys and compounds 266 180-5

[11] Fanfoni M and Tomellini M 1996 Physical Review B 54 9828-33

[12] Castro M, Dominguez-Adame F, Sanchez A and Rodriguez T 1999 Applied Physics Letters 75 $2205-7$

[13] Clavaguera-Mora M T, Clavaguera N, Crespo D and Pradell T 2002 Progress in Materials Science 47 559-619

[14] Shepilov M P 2004 Glass Physics and Chemistry 30 291-9

[15] Weinberg M C 1999 Journal of Non-Crystalline Solids 255 1-14

[16] Pineda E, Pradell T and Crespo D 2002 Philosophical Magazine A 82 107-21

[17] Pineda E and Crespo D 2003 Journal of Non-Crystalline Solids 317 85-90

[18] Tomellini M, Fanfoni M and Volpe M 2000 Physical Review B 62 11300-3

[19] Weinberg M and Kapral R 1989 J. Chem. Phys. 91 7146-52

[20] Birnie III D P and Weinberg M C 1995 J. Chem. Phys. 103 3742-46

[21] Pusztai T and Granasy L 1998 Physical Review B 57 14110-8

[22] Yu G, Lee S T and Lai J K L 1998 Physical Review B 57 13573-82

[23] Axe J D and Yamada Y 1986 Physical Review B 34 1599-606

[24] Meijering J L 1953 Philips Res. Rep. 8 270-90

[25] Almansour A, Matsugi K, Hatayama T and Yanagisawa O 1996 Materials Transactions JIM 37 1595-601

[26] Pineda E, Pradell T, Crespo D, Clavaguera N and Clavaguera-Mora M T 2001 Journal of NonCrystalline Solids 287 92-5

[27] Riedel M R and Karato S 1996 Geophys. J. Int. 125 397-414

[28] Pineda E and Crespo D 1999 Physical Review B 60 3104-12

[29] Pineda E, Bruna P and Crespo D 2004 Philosophical Magazine 84 2023-39

[30] Boots B N 1982 Metallography 15 53-62

[31] Fischer R A and Miles R E 1973 Math. Biosci. 18 335-50

[32] Stoyan D, Kendall W S and Mecke J 1989 Stochastic geometry and its application (Berlin: Akademie-Verlag Berlin) Chap. 10

[33] Gilbert E N 1962 The Annals of Mathematical Statistics 33 958-72

[34] Kiang T 1966 Zeitschrift fur Astrophisik 64 433-9

[35] Weaire D, Kermode J P and Wejchert J 1986 Philosophical Magazine B. 53 L101-5

[36] Kumar S, Kurtz S K, Banavar J R and Sharma M G 1992 Journal of Statistical Physics 67 523-51

[37] Moller J 1992 Adv. Appl. Probab. 24 814-44

[38] Mulheran P A 1992 Philosophical Magazine Letters 66 219-24

[39] Garrido V and Crespo D 1997 Physical Review E 56 2781-92

[40] Pineda E, Bruna P and Crespo D 2004 Physical Review E 70 066119-(1-8) 


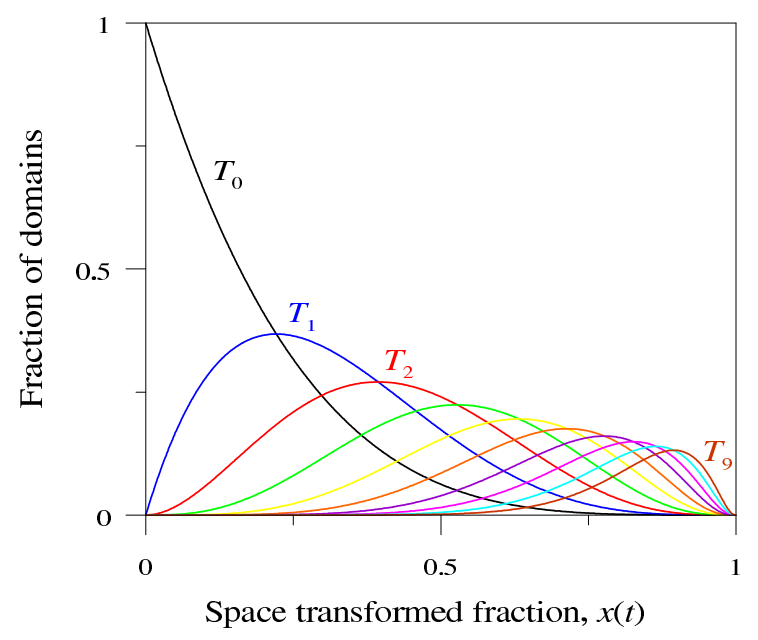

Figure 1. Temporal evolution of the $T_{k}(t)$ functions with $k$ going from 0 to 9 .

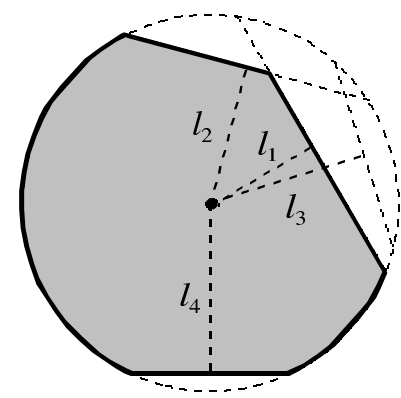

Figure 2. Possible collision configuration of a domain with 4 extended collisions at normalized times $l_{1}, l_{3}, l_{3}$ and $l_{4}$.

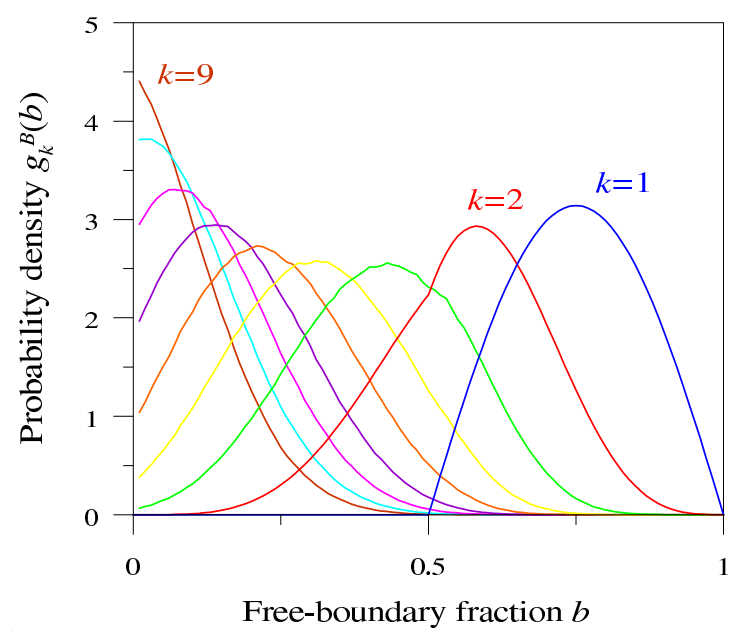

Figure 3. Time-invariant functions $g_{k}^{B}(b)$ with $k$ going from 1 to 9 . 


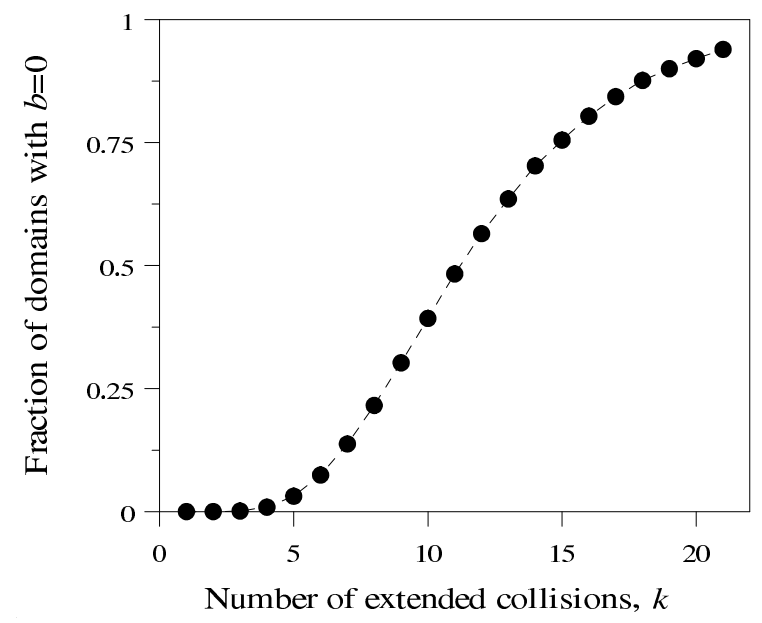

Figure 4. Fraction of completely blocked domains as a function of the number $k$ of extended collisions.

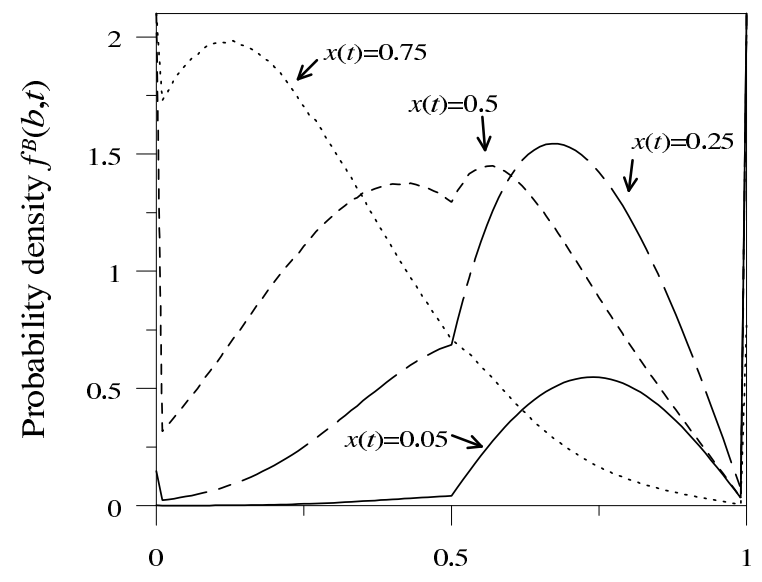

Free-boundary fraction $b$

Figure 5. Total free-boundary fraction probability density function at different stages of the transformation.

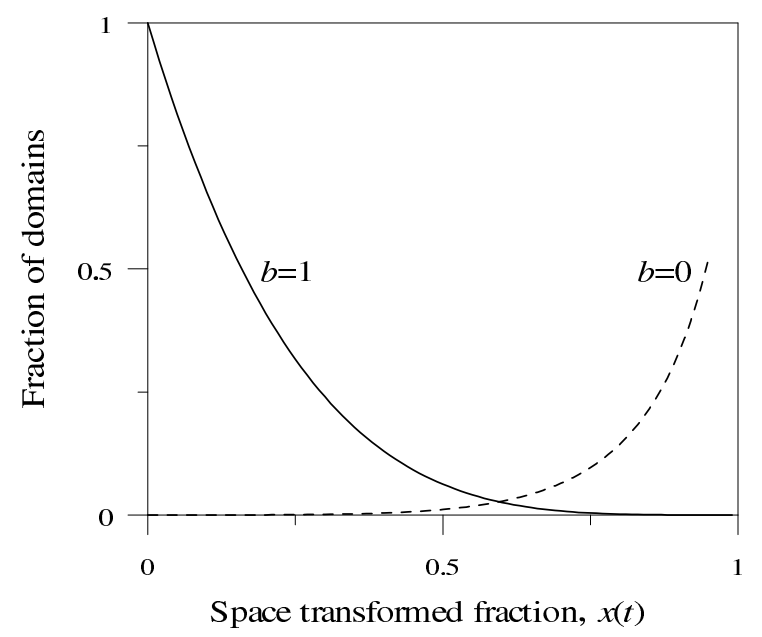

Figure 6. Temporal evolution of the probability of finding a domain without any collision (solid line) and a domain completely blocked by collisions (dashed line). 


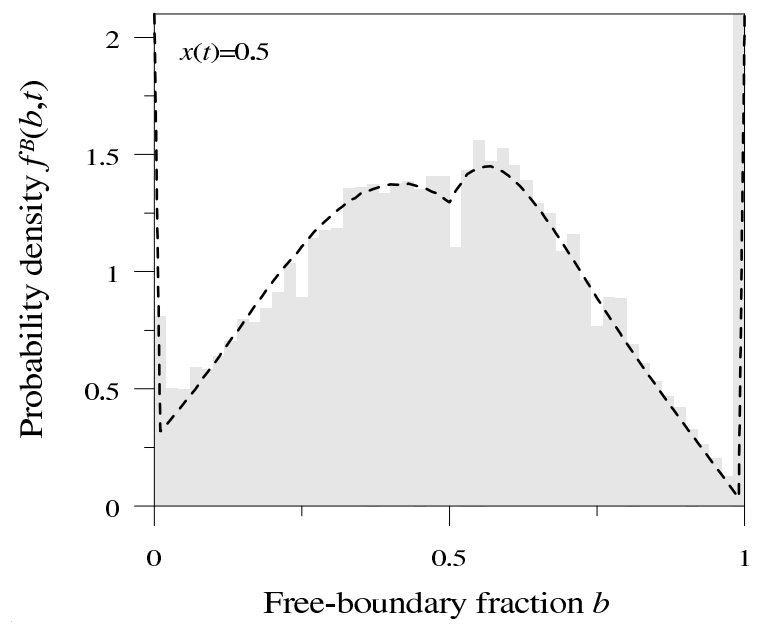

Figure 7. Calculated $f^{B}(b, t)$ at $x(t)=0.5$ compared with the result of stochastic simulations.

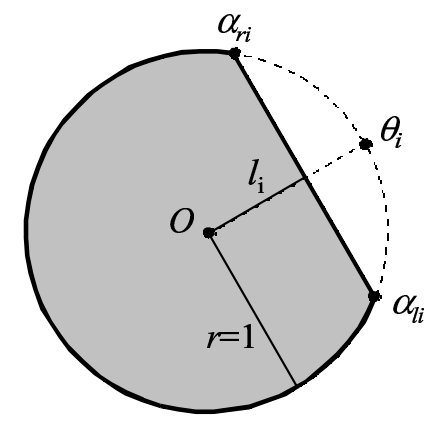

Figure 8. Normalized domain with a single collision at $\left(l_{i}, \theta_{i}\right)$.

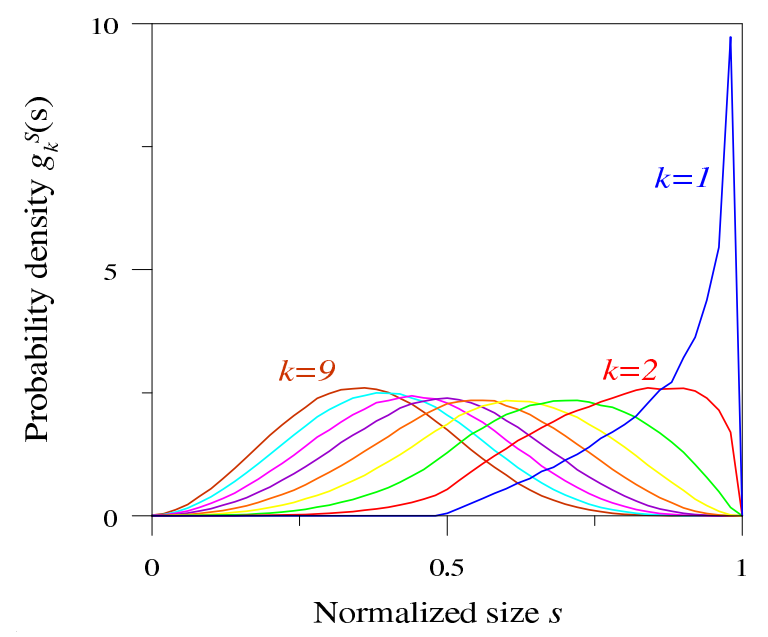

Figure 9. Time-invariant functions $g_{k}^{S}(s)$ with $k$ going from 1 to 9 . 


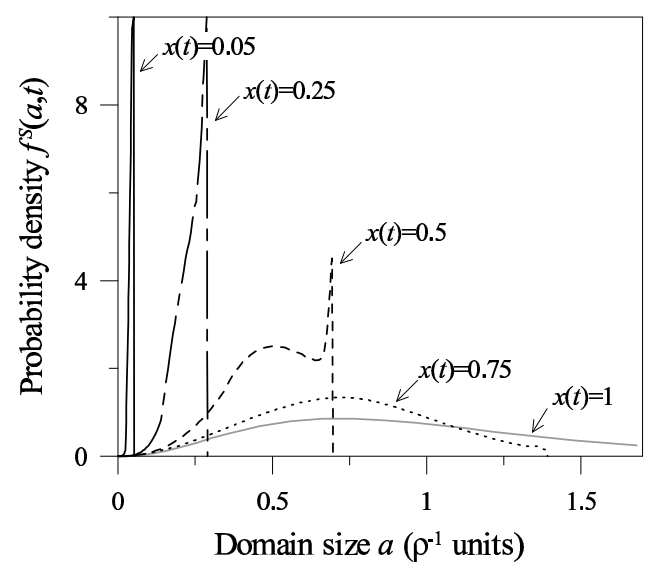

Figure 10. Total domain size probability density function at different stages of the transformation.

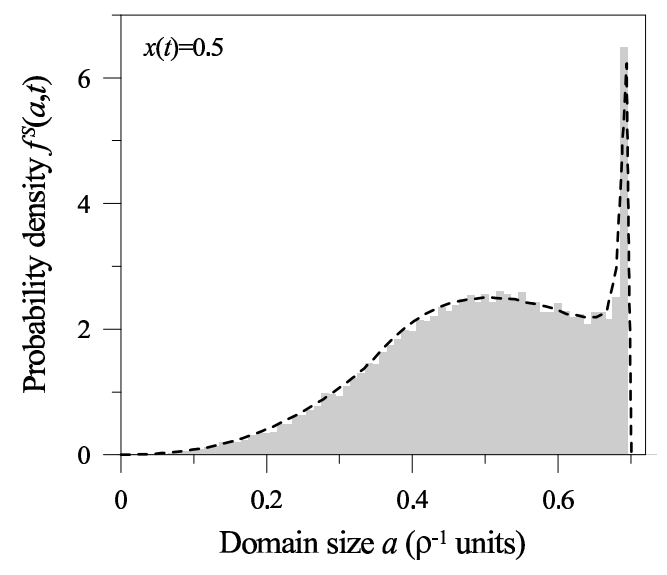

Figure 11. Calculated $f^{S}(s, t)$ at $x(t)=0.5$ compared with the result of stochastic simulations. 